\title{
Cyclic Nucleotides Mediate an Odor-evoked Potassium Conductance in Lobster Olfactory Receptor Cells
}

\author{
W. C. Michel ${ }^{1}$ and B. W. Ache ${ }^{1,2}$ \\ 'Whitney Laboratory and 'Departments of Zoology and Neuroscience, University of Florida, St. Augustine, Florida 32086
}

\begin{abstract}
Odors activate at least two distinct transduction pathways in lobster olfactory receptor cells that, respectively, excite and inhibit the cell. Data presented suggest that odors selectively activate the inhibitory conductance through the second messenger CAMP. Not all cells support both odorevoked excitatory and inhibitory conductances; in the current investigation, about $50 \%$ of the cells tested were inhibited by odors. In the majority of cells that, as a group, support an inhibitory response to odor stimulation, activation of adenylate cyclase with forskolin or inhibition of phosphodiesterase activity with 3-isobutyl-1-methylxanthine (IBMX) elicits an outward current with a time course similar to that of odor-evoked outward currents. The membrane-permeant cyclic nucleotide analogs 8-Br-cAMP and 8-Br-cGMP have a similar effect. Forskolin and IBMX enhance the magnitude of odor-evoked outward currents when the drug and the odor are copresented to the cell. In contrast, these same drugs have little or no effect on cells that, as a group, fail to support an inhibitory response to odor stimulation. This study provides the first direct evidence implicating CAMP in olfactory transduction in an invertebrate and contrasts with similar studies in vertebrates that have implicated CAMP as a second messenger mediating excitation.
\end{abstract}

It is generally accepted that the initial step in olfactory transduction involves odorant binding to specific membrane-associated, G-protein-coupled receptor proteins and the subsequent activation of one or more transduction pathways (reviewed by Anholt, 1991; Firestein, 1991). The most thoroughly studied of these second messenger pathways is the adenylate cyclase (AC) pathway in vertebrates. Odors stimulate cAMP synthesis (Pace et al., 1985) and do so sufficiently fast to precede the electrical response (Breer et al., 1990). Cyclic nucleotides were initially shown to gate directly a ciliary cation conductance (Nakamura and Gold, 1987) that subsequent whole-cell recordings link to odor stimulation. Intracellular cAMP elicits macroscopic currents similar to odor-evoked currents (Kurahashi, 1990; Firestein et al., 1991a). Apical application of membrane-permeant analogs of cyclic nucleotides, a specific AC activator, or a phosphodiesterase (PDE) inhibitor increases the rate of action po-

\footnotetext{
Received Feb. 20, 1992; revised May 6, 1992; accepted May 12, 1992.

We thank L. Van Ekeris for technical assistance and L. Milstead for help preparing the figures. This work was supported by ONR Grant N00014-90-J-1566 and NSF Grant BNS 88-10261.

Correspondence should be addressed to Dr. William C. Michel, University of Utah School of Medicine, Department of Physiology, 410 Chipeta Way, Salt Lake City, UT 84108

Copyright @ 1992 Society for Neuroscience $0270-6474 / 92 / 123979-06 \$ 05.00 / 0$
}

tential discharge in frog olfactory receptor neurons (ORNs) (Frings and Lindemann, 1991). In tiger salamander ORNs, odors and a PDE inhibitor activate a macroscopic current with similar kinetics (Firestein et al., 1991a). Unitary conductances that can be activated in cell-attached patches by odor or 8-bromo-cGMP (Firestein et al., 1991b) are directly gated by cyclic nucleotides in inside-out patches (Zufall et al., 1991). Indeed, olfactoryspecific AC (Bakalyar and Reed, 1990) and cAMP-gated cation channel (Dhallan et al., 1990; Ludwig et al., 1990) have been identified and sequenced.

Attempts to implicate cAMP as an olfactory second messenger in invertebrates have been limited to insects (Breer et al., 1990; Ziegelberger et al., 1990; Zufall and Hatt, 1991) and the spiny lobster (McClintock et al., 1989) but have yielded negative results, leading to the possibility that cAMP is an olfactory second messenger exclusively in vertebrates. In lobster olfactory (aesthetasc) receptor cells, odors activate inhibitory as well as excitatory currents (McClintock and Ache, 1989; Michel et al., 1991). These opposing, odor-activated currents are the result of increases in distinct membrane conductances (Schmiedel-Jacob et al., 1990; Michel et al., 1991), indicating that lobster olfactory receptor cells can support at least two different transduction pathways. Emerging evidence (Fadool et al., 1991) suggests that the excitatory pathway is mediated by inositol trisphosphate $\left(\mathrm{IP}_{3}\right)$, but the second messenger, if any, mediating the inhibitory pathway in the lobster is unknown. Earlier, we reported that forskolin and 3-isobutyl-1-methylxanthine (IBMX) stimulated production of CAMP in the outer dendritic segments of lobster olfactory receptor cells, but were unable to demonstrate odorstimulated production of cAMP (McClintock et al., 1989). We now report whole-cell data consistent with the hypothesis that cAMP selectively mediates the inhibitory pathway. The present finding can be reconciled with the earlier result in that odors vary in their ability to activate the two conductances, and the original test battery failed to include odors likely to activate the inhibitory pathway. Our results indicate that cAMP as an olfactory second messenger is not restricted to vertebrates and imply that cAMP-mediated transduction can be colocalized with at least one other transduction pathway, possibly mediated by $\mathrm{IP}_{3}$, in the same olfactory receptor cell.

\section{Materials and Methods}

Preparation. Adult specimens of the Caribbean spiny lobster, Panulirus argus, were obtained in the Florida Keys and maintained in flow-through aquaria on a mixed diet of squid, fish, and shrimp. The olfactory organ, an organized tuft of sensilla (aesthetascs) located on the lateral filament of the antennule, was excised into cold saline and cut into sections approximately $1 \mathrm{~mm}$ long, containing four or five annuli. Each section was split longitudinally, and the hemisection containing the olfactory sensilla was enzymatically cleaned with L-cysteine-activated papain 


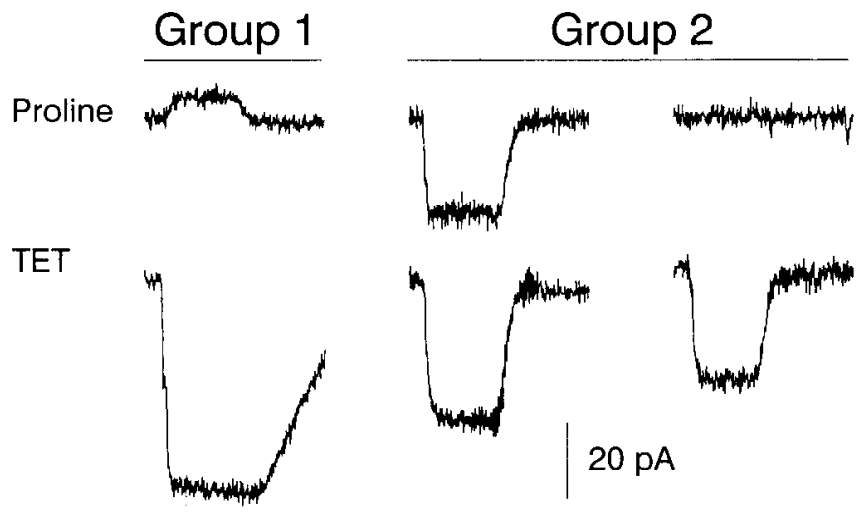

Saline
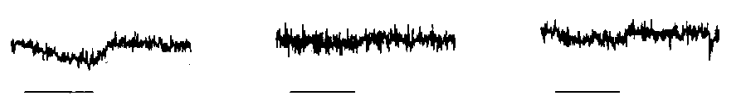

Figure 1. Current records of three olfactory receptors cells illustrate the patterns of responsiveness used to classify receptor cells tentatively into two groups. Group 1 cells were excited by TET and inhibited by proline, Group 2 cells were excited by TET but were tentatively classified as not supporting an inhibitory current if proline elicitcd an cxcitatory (inward) current (left) or if it was nonstimulatory (right). Each cell was voltage clamped at $-60 \mathrm{mV}$. The bar below each series of traces denotes $5 \mathrm{sec}$ of odor exposure.

(Sigma type IV, $0.25 \mathrm{mg} / \mathrm{ml}, 25 \mathrm{~min}$ ) and trypsin (Sigma type IX, 1 $\mathrm{mg} / \mathrm{ml}$ in calcium-free saline, $25 \mathrm{~min}$ ) and then rinsed and held in lobster saline at room temperature $\left(19-22^{\circ} \mathrm{C}\right)$. Such preparations were viable for 8-12 hr. Individual preparations were inserted into a slot molded into Sylgard elastomer, which formed the bottom of a combined recording bath/rapid olfactometer. A tight seal between the preparation and the elastomer isolated the bath from a lower flow-through compartment into which the olfactory sensilla projected. Details of the recording bath/olfactometer have been presented elsewhere (Michel et al., 1991). Odorants could be transiently (4-5 sec in the present investigation) and selectively presented to the olfactory sensilla without altering the saline bathing the receptor cell somata in the recording bath. In contrast to older olfactometers, which required several seconds to reach peak odor concentration, peak concentrations were achieved in $<100$ msec in the rapid olfactometer.

Recording protocol. Whole-cell current- or voltage-clamp recordings were achieved by forming gigaohm seals on the soma of olfactory receptor cells with borosilicate glass patch electrodes (Boralex, Dynalabs) with tip diameters of approximately $1 \mu \mathrm{m}$ (bubble number, ca. 5; Mittman et al., 1987). The electrodes were coated with Sylgard to reduce stray capacitance in most, but not all, recordings. The signals were amplified by a patch amplifier (Dagan 3900A), displayed on a digital oscilloscope (Nicolet 4094), and stored in digital form on an IBMcompatible AT computer using pCLAMP software (Axon Instruments, Inc.). The odor sensitivity of a neuron was established by challenging the cell with a complex odor mixture, TetraMarin (TET; see below) and recording a depolarization or inward current concomitant with the time course of the odor. Proline, the odorant that most effectively activated the inhibitory conductance in an earlier investigation (Michel et al., 1991), was then used to identify tentatively the subgroup of cells also supporting inhibitory conductances. Pharmacological agents targeting the adenylate cyclase system were presented in the same manner as were odors, either by themselves or together with an odor.

Solutions. The composition of the lobster saline was (in mM) 460 $\mathrm{NaCl}, 13 \mathrm{KCl}, 13 \mathrm{CaCl}_{2}, 10 \mathrm{MgCl}_{2}, 1.7$ glucose, 3 HEPES $(N$-2-hydroxyethylpiperazine- $N$-2-ethanesulfonic acid), $\mathrm{pH}$ 7.4. The composition of patch solution was (in $\mathrm{mM}$ ) $140 \mathrm{~K}$-acetate, $30 \mathrm{KCl}, 1 \mathrm{CaCl}_{2}$, 696 glucose, 11 EGTA (ethylene glycol bis- $N, N, N^{\prime}, N^{\prime}$-tetra-acetic acid), 10 HEPES, pH 7.0 (with $1 N \mathrm{NaOH}$, ca. $30 \mathrm{mM} \mathrm{Na}$ ). One micromolar 3-isobutyl-1-methylxanthine (IBMX), 8-bromoguanosine $3^{\prime}-5^{\prime}$-cyclic monophosphate (8-Br-cGMP), and 8-bromoadenosine $3^{\prime}-5^{\prime}$-cyclic monophosphate (8-Br-cAMP) were prepared fresh daily. Forskolin $(0.05$ $\mathrm{M})$ was dissolved in dimethyl sulfoxide (DMSO), maintained frozen until use, and diluted 1000-fold with saline before use.
Stock odorants were (1) an aqueous extract of the commercial fish food TET (TetraWerke, Melle, Germany), made by mixing $2 \mathrm{gm}$ of dry flakes in $60 \mathrm{ml}$ of saline, centrifuging the suspension at $1400 \times \mathrm{g}$, and filtering it through Whatman no. 3 filter paper, and (2) $10 \mathrm{mM}$ proline

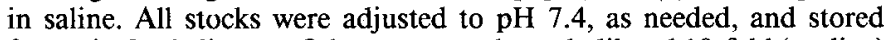
frozen in $5 \mathrm{ml}$ aliquots. Odorants were thawed, diluted 10 -fold (proline) or 100-fold (TET) with saline, and held on ice until use, daily. The concentrations of proline and TET were selected based on earlier studies that showed these doses elicit nearly saturating excitatory and inhibitory responses from the majority of cells. All chemicals were obtained from Sigma Chemical, St. Louis, $\mathbf{M}^{\mathrm{n}}$

\section{Results}

For the purpose of presenting the data, the ORNs were divided into two groups based on their responses to the amino acid proline (Fig. 1), the most effective of the odorants tested in an earlier study for activating the inhibitory conductance (Michel et al., 1991). Group $1(n=53)$ consisted of cells excited by TET and inhibited by proline. Group $2(n=53)$ consisted of cells excited by TET and not inhibited by proline, that is, cells that were either excited by or insensitive to proline. To facilitate the comparison of the effects of drugs across ORNs, the responses of any one cell to the drugs were standardized to the magnitude of the response of that cell to odors [TET (group 2) or proline (group 1), as appropriate]. The standardized responses were then averaged for all cells on which a particular drug was tested. This metric, of course, masks the absolute magnitude of the responses. The two groups of cells, however, did not differ in their capacity to respond to odors since the average magnitude of the response to TET for group 1 and group 2 cells was $-25.4 \pm$ $5.1 \mathrm{pA}( \pm \mathrm{SEM} ; n=23)$ and $-28.3 \pm 2.8 \mathrm{pA}( \pm \mathrm{SEM} ; n=36)$, respectively. As previously noted (Michel et al., 1991), the magnitude of the odor-evoked outward current was small, averaging $4.5 \pm 0.6 \mathrm{pA}( \pm \mathrm{SEM} ; n=23$ group 1 cells $)$. The scheme of classifying cells as not supporting an inhibitory conductance based solely on their response to proline is admittedly tentative since we know that other odorants also activate outward currents (Michel et al., 1991). It is intended to do no more than allow us to contrast clearly the pharmacological effects of drugs targeting specific parts of the AC pathway on odor-evoked excitatory and inhibitory currents.

\section{Effect of drugs targeting the AC pathway on group 1 cells}

Activation of $A C$. Forskolin, a membrane- permeable activator of $A C$, was used to establish the presence of the synthetic enzyme for cAMP. Forskolin $(50 \mu \mathrm{M})$ elicited an outward current in 19 (70\%) of 27 group 1 cells tested (Fig. 2). The time course of the forskolin-evoked current mimicked that of the odor-evoked outward current. Of the remaining cells, six were not stimulated by forskolin and two were weakly excited. No response could be detected to the concentration of DMSO used as a carrier of the forskolin (data not shown). The average response to forskolin was $48 \%$ of the response to proline and was significantly greater (paired $t$ test, $p<0.05$ ) than the response to the saline control (Fig. 3).

Role of PDE in odor-activation. If AC mediates the inhibitory conductance, a cyclic nucleotide PDE should be present to terminate the response by degrading cAMP. Assuming a basal AC activity, blocking PDE activity with the membrane-permeant PDE inhibitor IBMX would be expected to mimic the effect of an odor. IBMX (1 mM) elicited an outward current in $26(79 \%)$ of 33 group 1 cells tested (Fig. 2; also see Fig. 4). The time course of the IBMX-evoked outward current mimicked that of the odor-evoked outward current. The remaining cells were either 


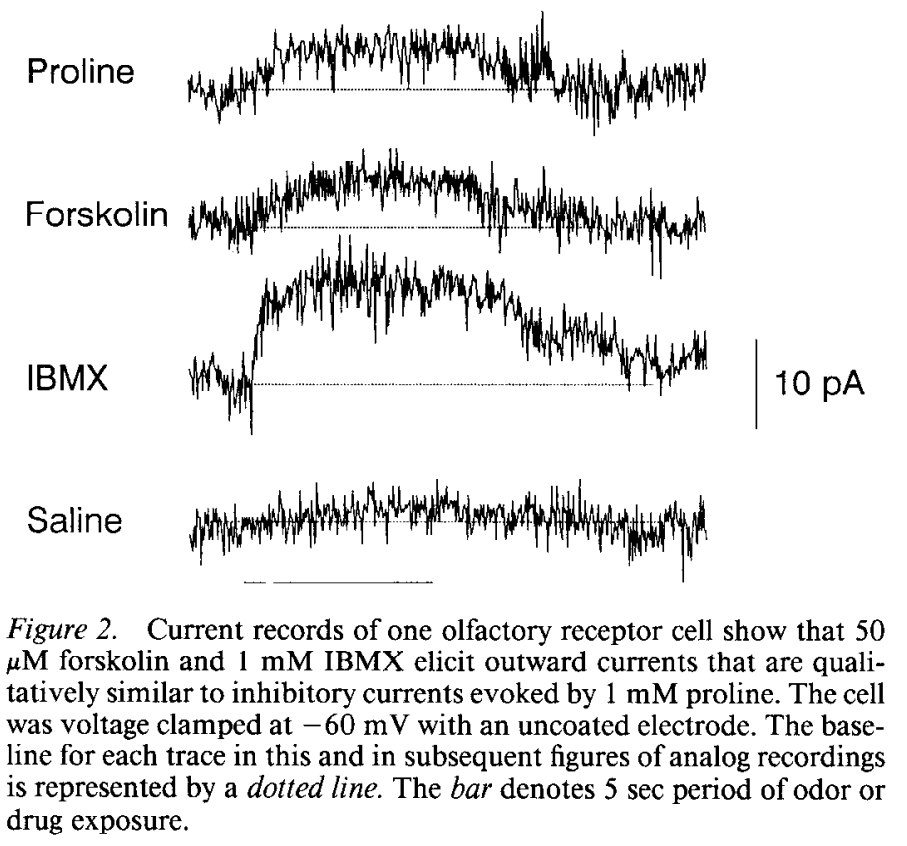

weakly excited $(n=4)$ or not stimulated $(n=3)$ by IBMX. The average magnitude of the IBMX response was $85 \%$ of the proline response and significantly greater (paired $t$ test, $p<0.05$ ) than the response to the saline control (Fig. 3).

If the basal AC activity is sufficient to activate the inhibitory conductance fully, then a mixture of forskolin and IBMX should elicit a response no larger than the response to IBMX alone. A mixture of forskolin and IBMX elicited a outward current in all of six cells tested. The average magnitude of the response to the mixture of forskolin and IBMX was $173 \%$ of the response to proline and larger than the response to IBMX alone (average magnitude $=132 \%$ for these cells) (Fig. 3).

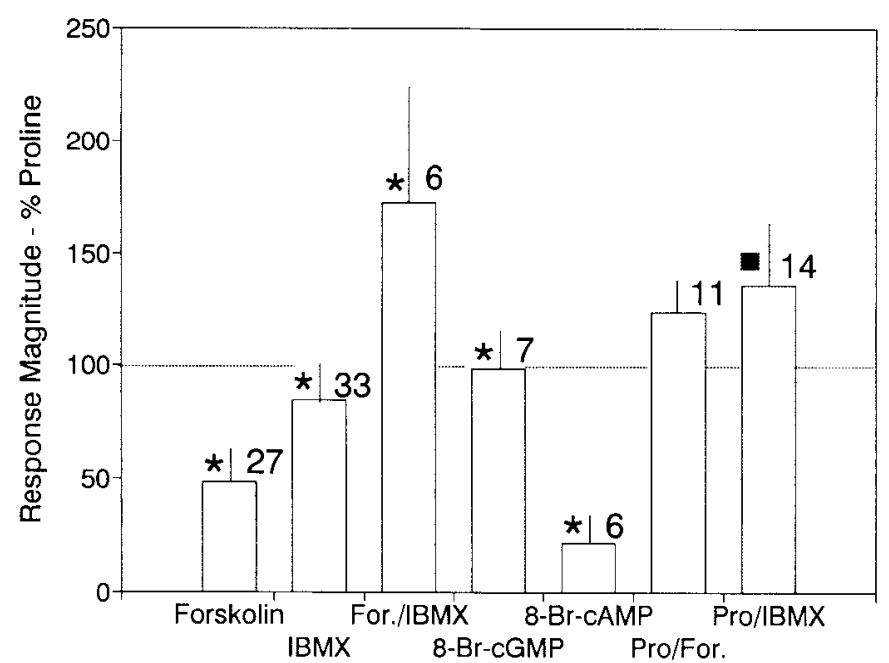

Figure 3. Average $( \pm$ SEM) effect of drugs targeting the AC pathway on dendritic currents for group 1 cells. The response of each cell to each drug is expressed as a percentage of the proline-evoked response $(100 \%$, dotted line). An asterisk indicates that the drug-evoked response is significantly greater than the control response (paired $t$ test, $p<0.05$ ). The responses to the mixtures of proline and forskolin or proline and IBMX were compared to the proline response $(\square$, significantly different, paired $t$ test, $p<0.05$ ). Numbers above each column correspond to the number of cells tested. Concentrations: forskolin, $50 \mu \mathrm{M}$; proline, IBMX, 8-BrcGMP, and 8-Br-cAMP, $1 \mathrm{mM}$.

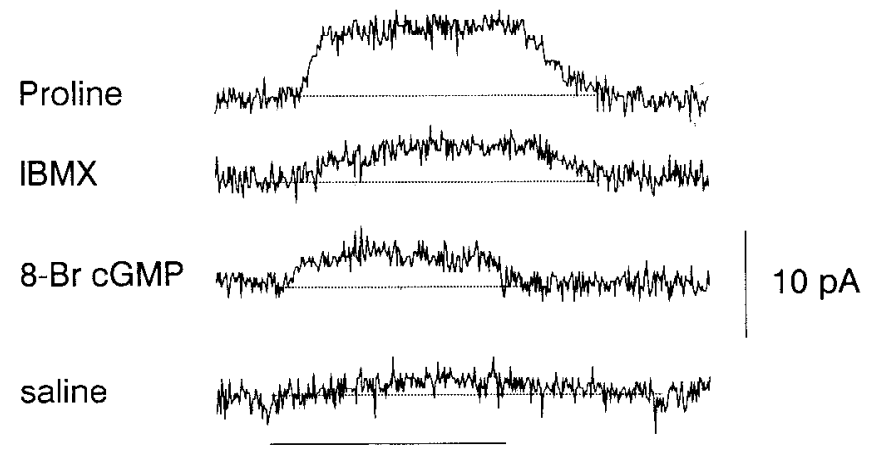

Figure 4. Current records of one olfactory receptor cell showing that inhibition of the PDE with IBMX or application of the membranepermeant cyclic nucleotide analog 8 -Br-cGMP elicited an outward current in receptor cells supporting odor-evoked inhibitory conductances. 8-Br-cAMP, also tested on this cell, did not elicit a measurable response. The bar denotes the $4 \mathrm{sec}$ period of odor or drug stimulation. Proline, IBMX, and 8-Br-cGMP, $1 \mathrm{mM}$.

Action of membrane-permeant cyclic nucleotide analogs. Attempts to activate the inhibitory conductance by introducing cAMP through the recording pipette failed, most probably due to the combination of the long diffusion distance from the soma to the dendrite (ca. $1 \mathrm{~mm}$; Grünert and Ache, 1988) and the rapid degradation of $\mathrm{cAMP}$ by the $\mathrm{PDE}(\mathrm{s})$. To circumvent these difficulties, we examined the effects of two stable, membranepermeant cyclic nucleotide analogs that could be applied directly to the dendrites. 8-Br-cGMP elicited an outward current in all of seven cells tested (Fig. 4). 8-Br-cAMP elicited an outward current in four of six ORNs. Of the two remaining cells, one was weakly excited, while the other was not stimulated by 8 $\mathrm{Br}$-cAMP. The kinetics of the 8-Br-cGMP-evoked currents mimicked the proline-evoked outward current for three of seven ORNs; for the four other cells tested, the onset of 8-Br-cGMP activated current mimicked the proline-evoked outward current but the analog-evoked current persisted longer (not shown). The average magnitude of the current elicited by 8 -Br-cAMP was only $20 \%$ of the magnitude of the 8 -Br-cGMP- and the odorevoked response (Fig. 3).

\section{Effect of drugs targeting the AC pathway on group 2 cells}

The actions of forskolin, IBMX, and the two permeant cyclic nucleotide analogs were also tested on group 2 cells to determine if transduction via cAMP was specific for the inhibitory pathway. Forskolin did not stimulate $15(65 \%)$ of 23 cells tested. The remaining cells were either inhibited $(n=4)$ or excited $(n$ $=4$ ) by forskolin. IBMX elicited no response from $25(57 \%)$ of 44 ORNs tested. Of the 19 remaining cells, 12 were inhibited and 7 were excited by IBMX. Three of eight cells tested with a mixture of forskolin and IBMX were inhibited, while the remaining five cells were not stimulated. Fifty percent of the cells tested with 8-Br-cGMP $(n=6)$ and 8-Br-cAMP $(n=6)$ were not stimulated by these drugs; the remaining cells were either weakly excited or inhibited. The average magnitudes of the responses to the drugs targeting the AC pathway for these cells (standardized to excitatory TET response) were not significantly different from control values and were less than $5 \%$ of the average magnitude of the odor-evoked response (Fig. 5). Several ORNs were excited by proline but inhibited by the pharmacological probes of the AC pathway, suggesting that activation of the AC pathway is not linked to a specific odorant (Fig. 6). 


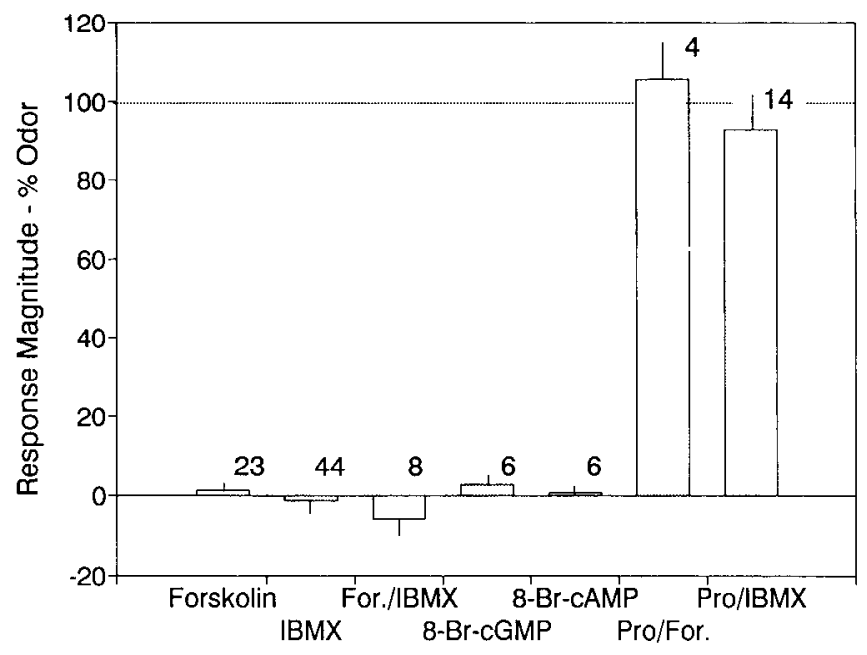

Figure 5. Bar graph showing the effects of drugs targeting the $\mathrm{AC}$ pathway on group 2 cells. The average $( \pm S E M)$ magnitudes of dendritic current evoked by drugs are expressed as a percentage of the TET-evoked excitatory response $(100 \%$, dotted line). Paired $t$ tests failed to identify any drug-evoked responses significantly different from the control responses or significant differences in the response to odor and forskolin or odor and IBMX compared to the response to odor alone. Numbers above each column correspond to the number of cells tested. Concentrations: forskolin, $50 \mu \mathrm{M}$; proline, IBMX, 8-Br-cGMP, and 8-Br-cAMP, $1 \mathrm{mM}$.

\section{Effect of drugs targeting the AC pathway on the response to proline}

The possible role of $\mathrm{AC}$ and PDE in odor-evoked transduction was further evaluated by determining the ability of forskolin and IBMX to alter the response to proline when the odor and the drug wcre copresented. For group 1 cells, the average magnitude of the response to a mixture of proline and forskolin and proline and IBMX was greater, $124 \%(n=11)$ and $136 \%(n=$ 14), respectively, than the average magnitude of the response to proline (Fig. 3). The response to the mixture of proline and IBMX was significantly greater than the response to proline alone (paired $t$ test, $p<0.05$ ). In contrast, for group 2 cells, the average response to a mixture of proline and forskolin or a mixture of proline and IBMX was not significantly different (paired $t$ test, $p>0.05), 106 \%(n=4)$, and 92\% $(n=14)$, respectively, from the average response to proline alone (Fig. 5).

\section{Discussion}

Collectively, our results suggest that the inhibitory conductance is activated through the second messenger, cAMP: (1) forskolin activates AC, (2) IBMX inhibits ongoing PDE activity, (3) membrane permeant cyclic nucleotide analogs mimic odor-evoked outward currents, (4) IBMX enhances odor-evoked outward currents, and (5) the action of the drugs is limited to cells that support an inhibitory response to odor stimulation.

Any one of the pharmacological probes could potentially bind to chemoreceptive sites and activate the inhibitory conductance as an odor rather than target the cAMP pathway per se. Indeed, this probably occurred in some percentage of cells. However, it is doubtful that three such structurally different compoundsforskolin (a plant alkaloid), IBMX (a methyl xanthine), and 8-Br-cGMP (a cyclic nucleotide analog)-generally acted as odors, particularly when tested and found to be effective on the same cell, as was the case in most experiments. For example,

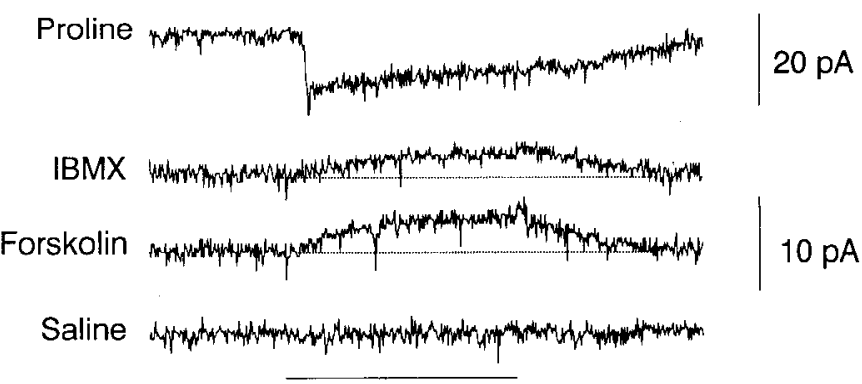

Figure 6. Current records of one olfactory receptor cell showing that drugs targeting the AC pathway may activate inhibitory currents in cells that were not inhibited by proline. Cell were held at $-60 \mathrm{mV}$. Forskolin, $50 \mu \mathrm{M}$; proline and IBMX, $1 \mathrm{mM}$. Bar denotes the $5 \mathrm{sec}$ period of odor or drug exposure.

both forskolin and IBMX were inhibitory for $72 \%$ of the group 1 cells tested with both drugs. In contrast, only one of seven group 2 cells strongly excited by forskolin was also excited by IBMX. We conclude, therefore, that cAMP is a second messenger in lobster ORNs, a conclusion that is consistent with the earlier finding that $\mathrm{AC}$ and $\mathrm{PDE}$ activity are present in the outer dendrites of lobster ORNs (McClintock et al., 1989).

Since 8-Br-cGMP was the more effective of the two membrane-permeant cyclic nucleotides we tested, the question arises as to whether CGMP and not CAMP is the physiologically active second messenger. Although single-channel recordings reveal that cGMP is more effective than cAMP in activating the cyclic nucleotide-gated conductances in amphibian ORNs (Nakamura and Gold, 1987; Zufall et al., 1991), there is abundant evidence that odors stimulate AC activity (reviewed by Anholt, 1991). To date, cGMP has not been implicated as a second messenger in olfactory transduction in either vertebrates or invertebrates. In one instance, cGMP was able to modulate a presumptive pheromone-activated channel in the outer dendrites of insect ORNs (Zufall and Hatt, 1991), but biochemical experiments failed to implicate cGMP in olfactory transduction in the same species (Ziegelberger et al., 1990). We conclude that the AC activity present is sufficient to account for the observed responses in lobster ORNs on the basis of the findings that forskolin (1) increases the concentration of cAMP in dendritic membranes (McClintock et al., 1989) and (2) mimics the odorevoked inhibitory conductance. As we have yet to test for guanylate cyclase activity in the lobster, we cannot exclude its involvement in the transduction process.

While odor-stimulated $\mathrm{AC}$ is not unique to lobster ORNs (see introductory remarks), the conductance subsequently activated is. In vertebrate ORNs, cAMP activates an inward (excitatory) current by directly gating a nonselective cation channel (Nakamura and Gold, 1987; Firestein et al., 1991b; Vodyanoy, 1991; Zufall et al, 1991). In lobster ORNs, in contrast, cAMP activates an outward (inhibitory) current through an increase in $g_{\mathrm{K}}$ (Michel et al., 1991). cAMP is known to activate or modulate $\mathbf{K}^{+}$ channels in other neurons (reviewed by Levitan, 1988), but heretofore has not been shown to do so in ORNs. In most instances where it has been studied, cAMP acts on $\mathrm{K}^{+}$channels indirectly, through activation of a kinase and subsequent phosphorylation of the channel. However, cAMP-gated $\mathrm{K}^{+}$channels have been reported recently in larval Drosophila muscle (Delgado et al., 1991), and preliminary data suggest that cAMP directly gates $\mathrm{K}^{+}$channel(s) in cultured lobster ORNs (D. A. Fadool and B. W. Ache, unpublished observations). Interest- 
ingly, olfactory and rod cyclic nucleotide-gated channels in vertebrates have a high degree of sequence homology and form a superfamily of ligand-gated channels with hydrophobicity plots that bear remarkable similarity to voltage-gated $\mathrm{K}^{+}$channels (Kaupp, 1991). While the mechanism by which cAMP activates the inhibitory conductance in lobster ORNs in situ remains to be determined, the possibility exists that the cAMP-gated potassium conductance in lobster ORNs represents yet another member of this family of ion channels.

The ability of forskolin and IBMX to elicit outward currents over essentially the same time course as do odors indicates the drugs must permeate the membrane rapidly and provides further insight into the underlying biochemistry. The rapid action of IBMX in the absence of an odor indicates basal AC activity, which would be consistent with our earlier determination that $\mathrm{AC}$ activity in the dendritic membranes in the absence of odors is approximately $4 \mathrm{pmol} / \mathrm{mg} / \mathrm{min}$ (McClintock et al., 1989). The basal AC activity presumably generates enough cAMP to activate the inhibitory conductance partially. This action presumably is countered by high PDE activity, which degrades the cAMP and keeps the inhibitory conductance from being continually active. Such a system would be able to respond rapidly to odors and may be a general feature of cAMP-mediated transduction in ORNs since inhibition of PDE activity by IBMX elicits macroscopic currents in tiger salamander ORNs (Firestein et al., 1991a) and since dendritic application of IBMX increases the rate of action potential discharge in frog ORNs (Frings and Lindemann, 1991).

What percentage of lobster ORNs support the inhibitory conductance? In two previous investigations, about $40 \%$ of the cells surveyed with a limited array of odors supported an inhibitory conductance, that is, the odors evoked an outward current or hyperpolarized the cell (McClintock and Ache, 1989; Michel et al., 1991). Given that only a limited array of odors has been (or can ever be) tested, the possibility exists that a greater proportion, possibly all, cells would support an inhibitory conductance were it possible to test all odors. If the inhibitory conductance is mediated strictly by cAMP, the present study can answer the question since the membrane-permeant drugs bypass the selectivity of the chemoreceptor(s) on the cell. $\Lambda$ s only $27 \%$ of the group 2 cells were identified as supporting an inhibitory conductance on the basis of their responses to IBMX, and the group 2 cells represented $50 \%$ of the cells tested in this study, only $13-14 \%$ more cells supported the inhibitory conductance than were identified by a single odor, proline. This finding suggests that the cAMP-mediated inhibitory pathway occurs in only a restricted subset of the receptor cell population.

The functional significance, if any, of having the cAMP-mediated transduction pathway expressed in only a subset of lobster ORNs is unknown. If mature lobster ORNs are continuously replaced, as are ORNs in vertebrates (Graziadei and MontiGraziadei, 1978) and snails (Chase and Rieling, 1986), the 50\% or so of lobster ORNs with the cAMP pathway may represent one developmental stage of what is otherwise a single type of ORN. In the absence of turnover, however, the apparent restricted expression of the cAMP pathway may be evidence for at least two functionally different subtypes of lobster ORNs. This would be a novel finding in olfaction since ORNs generally are not considered to be functionally diverse neurons beyond the diversity imposed by the receptor protein(s) they express. It is known, however, that vertebrate ORNs can be categorized into subgroups based on the presence or absence of various immunocytochemical markers (Akeson, 1988). Approximately $20-30 \%$ of the cells that were inhibited by proline appeared to be unresponsive to the drugs targeting the $\mathrm{AC}$ pathway. As a group, these cells responded only slightly to proline. The most likely explanation of these proline-sensitive, drug-insensitive cells is our inability to resolve small-magnitude drug-evoked responses ( $<4 \mathrm{pA}$ ) from noise (typically $2-4 \mathrm{pA}$ in our recording conditions), although the possibility of another transduction pathway mediating inhibition in these cells cannot be excluded.

Why did we fail to implicate cAMP in transduction in lobster ORNs previously, even though we could localize AC and PDE activity to the outer dendrites (McClintock et al., 1989)? First, proline and several other compounds now known to be effective stimuli for the inhibitory pathway (Michel et al., 1991) were not included in the limited battery of odors used to elevate levels of cAMP. Chemical analysis of TET (which was used as an odor in the earlier study) indicates that the mixture contains proline, but only at micromolar concentrations (Michel et al., 1991). Second, we were probably less able to resolve the small, hyperpolarizing changes in membrane potential in the earlier study that should have been evoked in at least some of the 42 cells tested with IBMX and/or forskolin, or 8-Br-cAMP. The probability of seeing the small changes in membrane potential expected from increasing $g_{\mathrm{K}}$ decreases as the rate of stimulus onset decreases, and the earlier study did not use the rapid olfactometer used in the present study (see Materials and Methods). Interestingly, 6 of the 42 cells did show a consistent increase in the magnitude of the excitatory receptor potential evoked by TET in the presence of (one of) the three pharmacological probes. If the six cells supported an inhibitory conductance, the prolonged exposure to the pharmacological probes may have increased the net excitation to TET by concurrently decreasing the driving force on the inhibitory conductance.

Our findings also suggest that cAMP does not mediate excitation in lobster ORNs. The responses of group 2 cells to the drugs were not significantly different from control responses. Although several cells were excited by either forskolin or IBMX, only one cell was identified that was excited by both forskolin and IBMX (data not shown). Finding that only a single cell was excited by both forskolin and IBMX is consistent with our earlier study, in which the same two drugs failed to depolarize any of 42 cells (McClintock et al., 1989). What, then, mediates excitation? Emerging evidence indicates that excitation is mediated by $\mathrm{IP}_{3}$ in cultured lobster ORNs (Fadool et al., 1991). If the cultured cells reflect lobster ORNs in situ, then cAMP and $\mathrm{IP}_{3}$ are not only dual olfactory second messengers in the lobster, as appears to be the case in rat (Boekhoff et al., 1990; Breer and Boekhoff, 1991) and catfish (Restrepo et al., 1990); they can also coexist in the same ORN.

Of particular interest is the functional significance of having opposing, odor-activated conductances in the same ORN. We propose that activating two transduction pathways compared to a single pathway increases the potential diversity of the output of the cell and, in doing so, more closely matches the response to the blend of components that constitute a particular odor. For example, two odors, identical in composition cxccpt for the concentration of an inhibitory component, would elicit identical responses from a cell supporting only the excitatory pathway, but distinct responses from a cell supporting opposing pathways. As odor recognition is presumed to require comparison of responses across the receptor cell population (Girardot and Derby, 1990), increasing the potential diversity of the output of single 
cells would enhance the across-cell pattern of responsc and better enable the lobster to distinguish similar odor mixtures.

\section{References}

Akeson RA (1988) Primary olfactory neuron subclasses. In: Molecular neurobiology of the olfactory system. Molecular, membranous and cytological studies (Margolis FL, Getchell TV, eds), pp 297-318. New York: Plenum.

Anholt RRH (1991) Odor recognition and olfactory transduction: the new frontier. Chem Senses 16:421-427.

Bakalyar HA, Reed RR (1990) Identification of a specialized adenylyl cyclase that may mediate odorant detection. Science 250:1403-1406.

Boekhoff I, Tareilus E, Strotmann J, Breer H (1990) Rapid activation of alternative second messenger pathways in olfactory cilia from rats by different odorants. EMBO J 9:2453-2458.

Breer H, Boekhoff I (1991) Odorants of the same odor class activate different second messenger pathways. Chem Senses 16:19-29.

Breer H, Boekhoff I, Tareilus E (1990) Rapid kinetics of second messenger formation in olfactory transduction. Nature 345:65-68.

Chase R, Rieling J (1986) Autoradiographic evidence for receptor cell renewal in the olfactory epithelium of a snail. Brain Res 384:232239.

Delgado R, Hidalgo P, Diaz F, Latorre R, Labarca P (1991) A cyclic AMP-activated $\mathrm{K}^{+}$channel in Drosophila larval muscle is persistently activated in dunce. Proc Natl Acad Sci USA 88:557-560.

Dhallan RS, Yau K-W, Schrader KA, Reed RR (1990) Primary structure and functional expression of a cyclic nucleotide-activated channel from olfactory ncurons. Nature 347:184-187.

Fadool DA, Michel WC, Ache BW (1991) G-proteins and inositolphospholipid metabolism implicated in odor response of cultured lobster olfactory neurons. Chem Senses 16:518-519.

Firestein S (1991) A noseful of odor receptors. Trends Neurosci 14: 270-272.

Firestein S, Darrow B, Shepherd GM (1991a) Activation of the sensory current in salamander olfactory receptor neurons depends on a $G$ protein-mediated cAMP second messenger system. Neuron 6:825835.

Firestein S, Zufall F, Shepherd GM (1991b) Single odor-sensitive channels in olfactory receptor neurons are also gated by cyclic nucleotides. J Neurosci 11:3565-3572.

Frings S, Lindemann B (1991) Current recording from sensory cilia of olfactory receptor cells in situ. I. The ncuronal response to cyclic nucleotides. J Gen Physiol 97:1-16.

Girardot M-N, Derby CD (1990) Peripheral mechanisms of olfactory discrimination of complex mixtures by the spiny lobster: no cell types for mixtures but different contributions of the cells to the across neuron patterns. Brain Res 513:225-236.

Graziadei PPC, Monti-Graziadei GA (1978) Continuous nerve cell renewal in the olfactory system. In: Handbook of sensory physiology, Vol 9 (Jacobson M, ed), pp 55-83. New York: Springer.
Grünert U, Ache BW (1988) Ultrastructure of the aesthetasc (olfactory) sensilla of the spiny lobster, Panulirus argus. Cell Tissue Res 251:95-103.

Kaupp UB (1991) The cyclic nucleotide-gated channels of vertebrate photoreceptors and olfactory epithelium. Trends Neurosci 14:150157.

Kurahashi T (1990) The response induced by intracellular cyclic AMP in isolated olfactory receptor cells of the newt. J Physiol (Lond) 430: 355-371.

Levitan IB (1988) Modulation of ion channels in neurons and other cells. Annu Rev Neurosci 11:119-136.

Ludwig J, Margalit T, Eismann E, Lancet D, Kaupp UB (1990) Primary structure of cAMP-gated channel from bovine olfactory epithelium. FEBS Lett 270:24-29.

McClintock TS, Ache BW (1989) Hyperpolarizing receptor potentials in lobster olfactory receptor cells: implications for transduction and mixture suppression. Chem Senses 14:637-647.

McClintock TS, Schütte K, Ache BW (1989) Failure to implicate cAMP in transduction in lobster olfactory receptor cells. Chem Senses 14:817-827.

Michel WC, McClintock TS, Ache BW (1991) Inhibition of lobster olfactory receptor cells by an odor-activated potassium conductance. J Neurophysiol 65:446-453.

Mittman S, Flaming DG, Copenhagen DR, Belgum JH (1987) Bubble pressure measurement of micropipet tip outer diameter. J Neurosci Methods 22:161-166.

Nakamura T, Gold GH (1987) A cyclic nucleotide-gated conductance in olfactory receptor cilia. Nature 325:442-444.

Pacc U, Hanski E, Salomon Y, Lancet D (1985) Odorant-sensitive adenylate cyclase may mediate olfactory reception. Nature $316: 255-$ 258.

Restrepo D, Miyamoto T, Bryant BP, Teeter JH (1990) Odor stimuli trigger influx of calcium into olfactory neurons of the channel catish. Science 249:1166-1168.

Schmiedel-Jakob I, Michel WC, Anderson PAV, Ache BW (1990) Whole cell recording from lobster olfactory receptor cells: multiple ionic bases for the receptor potential. Chem Senses 15:397-405.

Vodyanoy V (1991) Cyclic AMP-sensitive ion channels in olfactory receptor cells. Chem Senses 16:175-180.

Ziegelberger G, Van den Berg MJ, Kaissling K-E, Klumpp S, Schultz. JE (1990) Cyclic GMP levels and guanylate cyclase activity in pheromone-sensitive antennae of the silkmoths Antheraea polyphemus and Bombyx mori. J Neurosci 10:1217-1225.

Zufall F, Hatt H (1991) Dual activation of a sex pheromone-dependent ion channel from insect olfactory dendrites by protein kinase $\mathrm{C}$ activators and cyclic GMP. Proc Natl Acad Sci USA 88:8520-8524.

Zufall F, Firestein S, Shepherd GM (1991) Analysis of single cyclic nucleotide-gated channels in olfactory receptor cells. J Neurosci 11: 3573-3580. 